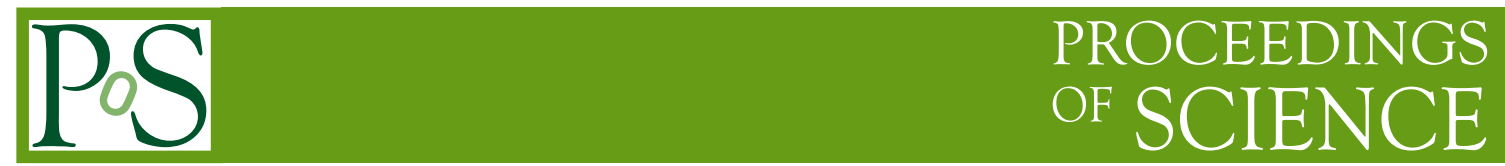

\title{
$\eta-\eta^{\prime}-$ glue Mixing from the Chiral Lagrangian
}

\author{
Vincent Mathieu* \\ Departament de Física Teòrica and Institut de Física Corpuscular, \\ Universitat de València-CSIC, E-46100 Burjassot (Valencia), Spain. \\ E-mail: vincent,mathieudumons.ac.be

\section{Vicente Vento} \\ Departament de Física Teòrica and Institut de Física Corpuscular, \\ Universitat de València-CSIC, E-46100 Burjassot (Valencia), Spain. \\ E-mail: vicente.ventodur.es
}

The $\eta-\eta^{\prime}$ mixing from the chiral Lagrangian is reviewed. It is shown how the Feldman-KrollStech ansatz can be derived from the chiral Lagrangian. The inclusion of the glueball is also discussed.

Light Cone 2010: Relativistic Hadronic and Particle Physics June 14-18, 2010

Valencia, Spain

\footnotetext{
* Speaker.
} 


\section{Introduction}

Guided by symmetry, the chiral Lagrangian is one of the most powerful tools to study the interaction of Goldstone bosons. The $\eta^{\prime}$ is not a Goldstone bosons because of the $U(1)$ axial anomaly but its inclusion in the chiral Lagrangian can be done using large $N$ arguments.

In this work, we rewrite the mass matrix in terms of physical quantities related to low energy constants which help us identify the relevant flavor basis well suited to deal with the mixing problem. In this basis, every unknown can be expressed (approximately) in term of four masses $M_{\pi}$, $M_{K}, M_{\eta}, M_{\eta^{\prime}}$, which become our only inputs.

The relevance of flavor was identified in the celebrated Feldmann-Kroll-Stech (FKS) formalism [四]. To derive their mass matrix, the authors of ref. [W] assumed that the decay constants follow the particle state mixing pattern. Under this assumption, they extracted a theoretical determination of the mixing angle.

Without the need for a decay constants pattern, we derive from the chiral Lagrangian a mass matrix in the flavor basis which compares well with FKS and shows how the assumption of ref. [四] influences the mass matrix.

\section{The chiral Lagrangian at $\mathscr{O}\left(p^{2}\right)$}

Let us briefly review what is known from chiral Lagrangians at leading order. In the large $N$ limit, the relevant degrees of freedom are the nine Goldstone bosons of the symmetry breaking $U(3)_{L} \otimes U(3)_{R} \rightarrow U(3)_{V}$. We collect the Goldstone mesons in a nonlinear parametrization $U=$ $\exp (i \sqrt{2} \pi / f)$ with $\pi=\pi^{a} \lambda_{a}$, with $\lambda_{0} \equiv 1_{3} \sqrt{2 / 3}$.

The chiral Lagrangian is constructed via an expansion in the momenta. At each order in $p^{2}$ we only retain retain the dominant term in the $1 / N$ expansion [[]]. Collecting all terms at $\mathscr{O}\left(p^{2}\right)$ and expanding the Lagrangian in the fields, one obtains

$$
\mathscr{L}^{\left(p^{2}\right)}=\frac{1}{2} \partial_{\mu} \pi^{a} \partial^{\mu} \pi^{b} \delta_{a b}-\frac{1}{2} B \pi^{a} \pi^{b}\left\langle\lambda_{a} \lambda_{b} \mathscr{M}\right\rangle-\frac{1}{2} \alpha_{0} \eta_{0}^{2} .
$$

The incorporation of the isosinglet $\eta_{0}$ induces a mixing with the $\eta_{8}$. The masses of the two physical states $\eta$ and $\eta^{\prime}$ are the eigenvalues of the mass matrix for the $\eta_{8}-\eta_{0}$ system

$$
\mathscr{M}_{80}^{2}=\frac{1}{3}\left(\begin{array}{cc}
4 m_{K}^{2}-m_{\pi}^{2} & -2 \sqrt{2}\left(m_{K}^{2}-m_{\pi}^{2}\right) \\
-2 \sqrt{2}\left(m_{K}^{2}-m_{\pi}^{2}\right) & 2 m_{K}^{2}+m_{\pi}^{2}+3 \alpha_{0}
\end{array}\right) .
$$

At this stage, $\alpha_{0}$ is an unknown parameter.

Furthermore, as we shall see, the diagonal mass matrix induces a privileged basis. For this it is useful to work in the flavor basis. For this purpose, we use the representation $\pi=\sqrt{2} \operatorname{diag}(u \bar{u}, d \bar{d}, s \bar{s})$.

We next introduce the fields $\eta_{s}=s \bar{s}$ and $\eta_{q}=(u \bar{u}+d \bar{d}) / \sqrt{2}$ (and the orthogonal counterpart of $\eta_{q},(u \bar{u}-d \bar{d}) / \sqrt{2}$ which decouples under isospin symmetry) allowing to rewrite the mass matrix as

$$
\mathscr{M}_{q s}^{2}=\left(\begin{array}{cc}
m_{\pi}^{2}+2\left(\alpha_{0} / N\right) & \left(\alpha_{0} / N\right) \sqrt{2} \\
\left(\alpha_{0} / N\right) \sqrt{2} & 2 m_{K}^{2}-m_{\pi}^{2}+\left(\alpha_{0} / N\right)
\end{array}\right) .
$$

However, with only one parameter $\alpha_{0}$ is not possible to reproduce the two physical masses [0]. 


\section{The chiral Lagrangian at $\mathscr{O}\left(p^{4}\right)$}

For our purposes, we are only interested in terms contributing to the kinetic part, the mass matrix and the decay constants. We restrict ourselves to terms involving only zero or two derivatives, and we only keep the leading order in $1 / N$. There are three terms of interest at $\mathscr{O}\left(p^{4}\right)$ and they involve a single trace over flavor [2]:

$$
\frac{f^{2}}{8}\left[-\frac{B}{\Lambda^{2}}\left\langle\mathscr{M} \partial_{\mu} \partial^{\mu} U^{\dagger}\right\rangle+\frac{B^{2}}{2 \Lambda_{1}^{2}}\left\langle\mathscr{M} U^{\dagger} \mathscr{M} U^{\dagger}\right\rangle+\frac{B}{2 \Lambda_{2}^{2}}\left\langle\mathscr{M} U^{\dagger} \partial_{\mu} U \partial^{\mu} U^{\dagger}\right\rangle\right]+\text { h.c.. }
$$

The three low energy constants enter the observables without a clear physical meaning. $\Lambda$ and $\Lambda_{2}$ induce a splitting between the $\pi$ and $K$ decay constants. $\Lambda_{1}$ and $\Lambda_{2}$ enter in the corrections to the mass matrix. Fitting their values on observables leads to a consistent $\eta-\eta^{\prime}$ scheme at $\mathscr{O}\left(p^{4}\right)[[]$ ].

We are interested in a more physical interpretation of the low energy constants and we aim at an analytical resolution of the mass matrix. We therefore would like to reduce the number of parameters since a two-by-two matrix gives us only two independent equations. To this end, we perform the rotation

$$
U \longrightarrow U^{\prime}=U-\frac{B}{2 \Lambda^{2}}\left(\mathscr{M}-U \mathscr{M}^{\dagger} U\right)
$$

Now, the low-energy constants appear in a more convenient way [3]. The wave-function renormalizations are simply proportional to the matrix of the decay constants. This matrix is proportional to $\left\langle\lambda_{a} \lambda_{b} \mathscr{M}\right\rangle$. Renormalizing the fields is easy in the case of a diagonal matrix since it only amounts to renomalizing the fields by a simple rescaling without any rotation. This is not the case in the $U(3)$ basis where the rotation is mandatory to brings us in the flavor basis where $\left\langle\lambda_{a} \lambda_{b} \mathscr{M}\right\rangle$ becomes diagonal. It is then more advantageous to start directly in the flavor basis. In the flavor basis the kinetic terms

$$
\frac{1}{2}\left(\frac{f_{q}}{f}\right)^{2} \partial_{\mu} \eta_{q} \partial^{\mu} \eta_{q}+\frac{1}{2}\left(\frac{f_{s}}{f}\right)^{2} \partial_{\mu} \eta_{s} \partial^{\mu} \eta_{s}
$$

does not present any mixing term. Moreover, since the kinetic energy and the decay constants follow the same pattern, the matrix of the decay constants is also diagonal. This avoids unwanted transition elements $J_{\mu}^{8}\left|\eta_{0}\right\rangle$. The particular structure of the mass matrix imposes then a privileged basis as claimed below and used in the FKS scheme [四].

The flavor decay constants can be easily expressed in term of the physical ones (in the $U(3)$ basis): $f_{q}^{2}=f_{\pi}^{2}$, and $f_{s}^{2}=2 f_{K}^{2}-f_{\pi}^{2}$.

The mass matrix then involves those physical decay constants. Introducing the parameter $y=f_{q} / f_{s}$, we can derive the mass matrix in a more convenient form

$$
\mathscr{M}_{q s}^{2}=\left(\begin{array}{cc}
M_{q q}^{2}+2 \alpha & \alpha y \sqrt{2} \\
\alpha y \sqrt{2} & M_{s s}^{2}+\alpha y^{2}
\end{array}\right)+\mathscr{O}\left(\frac{\alpha}{\Lambda^{2}}\right) .
$$

Assuming a negligible term $\mathscr{O}\left(\alpha / \Lambda^{2}\right)$ provides us analytical formulas, while the inclusion of this term leads to a unavoidable numerical procedure.

From (B.4) and neglecting the term $\mathscr{O}\left(\alpha / \Lambda^{2}\right)$ (or equivalently taking the limit $\Lambda \rightarrow \infty$ ), it is straightforward to extract the value of the parameters $y$ and $\alpha$ in function of the masses. Equating the determinant and the trace of (3.4) with the mass matrix matrix of the physical states, 
$\operatorname{diag}\left(M_{\eta}^{2}, M_{\eta^{\prime}}^{2}\right)$, we obtain

$$
\begin{aligned}
y^{2} & =2 \frac{M_{\eta}^{2} M_{\eta^{\prime}}^{2}-M_{s s}^{2}\left(M_{\eta}^{2}+M_{\eta^{\prime}}^{2}-M_{s s}^{2}\right)}{M_{\pi}^{2}\left(M_{\eta}^{2}+M_{\eta^{\prime}}^{2}-M_{\pi}^{2}\right)-M_{\eta}^{2} M_{\eta^{\prime}}^{2}}, \\
\alpha & =\frac{M_{\eta}^{2}+M_{\eta^{\prime}}^{2}-M_{\pi}^{2}-M_{s s}^{2}}{2+y^{2}}, \quad \sin 2 \varphi=\frac{2 \sqrt{2} \alpha y}{M_{\eta^{\prime}}^{2}-M_{\eta}^{2}} .
\end{aligned}
$$

We quoted the expression for the mixing angle in the flavor basis defined in a standard way by

$$
\left(\begin{array}{c}
\eta \\
\eta^{\prime}
\end{array}\right)=\left(\begin{array}{cc}
\cos \phi & -\sin \phi \\
\sin \phi & \cos \phi
\end{array}\right)\left(\begin{array}{l}
\eta_{q} \\
\eta_{s}
\end{array}\right)
$$

In the mass matrix (B.4), $M_{q q}$ and $M_{s s}$ are the unknown masses of the pseudoscalar $q \bar{q}$ and $s \bar{s}$ states. They can be related to physical masses by expressing them in the $U(3)$ basis. We obtain []]

$$
M_{q q}^{2}=M_{\pi}^{2}, \quad M_{s s}^{2}=2 M_{K}^{2}-M_{\pi}^{2}+\left(M_{K}^{2}-M_{\pi}^{2}\right)^{2}\left(\frac{2}{\Lambda_{1}^{2}}-\frac{1}{\Lambda_{2}^{2}}\right) .
$$

We are not able to relate $M_{s s}^{2}$ with the physical masses at $\mathscr{O}\left(p^{4}\right)$. We can approximate $M_{s s}^{2}$ by $2 M_{K}^{2}-M_{\pi}^{2}$ (exact only at $\mathscr{O}\left(p^{2}\right)$ ), the error on $M_{s s}$ being less than $5 \%$. This approximation coincides with the value used in the FKS paper [四].

We then obtain the value for the two parameters $y$ and $\alpha$ and a prediction for the physical quantities

$$
\frac{f_{K}}{f_{\pi}}=1.146, \quad \phi=41.40^{\circ} .
$$

Our calculated values (B.9) lie in the usual range $\left[40^{\circ}, 45^{\circ}\right]$ and are in agreement with the data on $\eta$ and $\eta^{\prime}$ decays. The phenomenological value for the ratio $f_{K} / f_{\pi}=1.193(0.009)$ [四] is also close to our predicted value.

The approximation $M_{s s}^{2}=2 M_{K}^{2}-M_{\pi}^{2}$ is only valid at leading order and receives a correction at $\operatorname{order} \mathscr{O}\left(p^{4}\right)\left[[]\right.$. The extra contribution reduces the value of the mixing angle and $f_{K} / f_{\pi}$ as shown in ref. [B]].

\section{Comparison with FKS Formalism}

We next compare the prediction of the chiral Lagrangian with the FKS results [四]. The basic hypothesis of the FKS formalism was the assumption that decay constants in the flavor basis follow the same mixing pattern (B.7) of the states

$$
\left(\begin{array}{ll}
f_{\eta}^{q} & f_{\eta}^{s} \\
f_{\eta^{\prime}}^{q} & f_{\eta^{\prime}}^{s}
\end{array}\right)=\left(\begin{array}{cc}
\cos \phi & -\sin \phi \\
\sin \phi & \cos \phi
\end{array}\right)\left(\begin{array}{cc}
f_{q} & 0 \\
0 & f_{s}
\end{array}\right) .
$$

Under this assumption, the mass matrix is derived from current algebra and reads

$$
\left(\begin{array}{cc}
M_{q q}^{2}+\frac{\sqrt{2}}{f_{q}}\left\langle 0\left|\frac{\alpha_{s}}{4 \pi} G \tilde{G}\right| \eta_{q}\right\rangle & \frac{1}{f_{s}}\left\langle 0\left|\frac{\alpha_{s}}{4 \pi} G \tilde{G}\right| \eta_{q}\right\rangle \\
\frac{\sqrt{2}}{f_{q}}\left\langle 0\left|\frac{\alpha_{s}}{4 \pi} G \tilde{G}\right| \eta_{s}\right\rangle & M_{s s}^{2}+\frac{1}{f_{s}}\left\langle 0\left|\frac{\alpha_{s}}{4 \pi} G \tilde{G}\right| \eta_{s}\right\rangle
\end{array}\right)
$$


Let us see how it arises from the chiral Lagrangian.

In our effective approach, the anomaly couples equally to each flavor before the renormalization of the fields. Indeed we have $\left(\partial^{\mu} A_{\mu}^{0}\right)_{\text {anomaly }}=f \alpha_{0}\left(\sqrt{2} \eta_{q}+\eta_{s}\right)$, which should be compare to $\left(\partial^{\mu} A_{\mu}^{0}\right)_{\text {anomaly }}=3 \alpha_{s} /(4 \pi) G_{\mu \nu}^{a} \tilde{G}_{a}^{\mu \nu}$. Rewriting $\mathscr{M}_{\mathrm{FKS}}^{2}$ in our notation, we get (in their paper [四], the authors denoted the anomaly by $a^{2}=\alpha$ )

$$
\mathscr{M}_{\mathrm{FKS}}^{2}=\left(\begin{array}{cc}
M_{q q}^{2}+2 \alpha & \alpha y \sqrt{2} \\
\alpha y \sqrt{2} & M_{s s}^{2}+\alpha y^{2}
\end{array}\right)
$$

We clearly see the equivalence of the mass matrices (4.2) and (B.4) at $\mathscr{O}\left(\alpha / \Lambda^{2}\right)$. The mass matrix is then symmetric and we do not need to impose the equality of the off diagonal terms by hand as in ref. [四].

The important point is that we did not use any assumptions of the mixing scheme for the decay constants in the derivation of (B.4). The hypothesis (4. field theory point of view and is not physically justified on general grounds. By comparing both approaches, we notice that the hypothesis on the decay constants pattern is not consistent with the chiral Lagrangian at next to leading order in the large $N$ limit since it amounts to send $\Lambda \rightarrow \infty$.

\section{Inclusion of the Glueball}

The inclusion of the glueball follow the same lines as the explicit breaking of chiral symmetry. The mass term breaks the chiral symmetry in the QCD Lagrangian. However, if we allow the mass term $\mathscr{M}$ to transform like $U^{\dagger}$, the mass term in the effective Lagrangian will mimic the breaking in the original Lagrangian.

The $U(1)_{A}$ transformation induces an extra term $\theta F_{\mu v} \tilde{F}^{\mu v}$ in the QCD Lagrangian. Accordingly, we allow $\theta$ to transform such that the combinaison $\eta_{0}+\theta$ remains invariant. $\theta$ couples to $F_{\mu v} \tilde{F}^{\mu v}$ and represents then a pseudoscalar purely gluonic field. We add a mass and a kinetic terms for the gluonic field and we obtain the action [[]]

$$
\mathscr{L}^{\left(p^{2}\right)}=\frac{f^{2}}{8}\left\langle\partial_{\mu} U^{\dagger} \partial^{\mu} U+B\left(m U^{\dagger}+U m^{\dagger}\right)\right\rangle-\frac{\alpha}{2}\left(\eta_{0}+\theta\right)^{2}-\frac{1}{2} m_{\theta}^{2} \theta^{2}+\frac{1}{2} \partial_{\mu} \theta \partial^{\mu} \theta
$$

The mass matrix involves three unknowns and can be solve analytically. But by imposing the physical masses for the $\eta$ and $\eta^{\prime}$ we cannot accommodate the decays [5]. However, if we allow the masses to differ slightly we find the optimal mixing scheme to be [6]

$$
\left(\begin{array}{ccc}
0.9709 & 0.2231 & -0.0872 \\
-0.2292 & 0.7590 & -0.6094 \\
-0.0698 & 0.6116 & 0.7881
\end{array}\right),
$$

with the corresponding to the values $\theta=-13.3^{\circ}, \varphi_{G}=4^{\circ}, \varphi=37.8^{\circ}$ and $M_{\eta}=523 \mathrm{MeV}, M_{\eta^{\prime}}=$ $1020, \mathrm{MeV}_{G}=1491 \mathrm{MeV}$. The third partner with a mass around $1500 \mathrm{MeV}$ could be associated to the $\eta(1405)$. However, a further analysis needs to be perform to confirm this hypothesis [ 6 ]. We note also that, introducing the gluonic state, allows to reconciliate all decays around a common value for the mixing angle $\theta \sim-15^{\circ}$ where the typical value obtain from processes involving the anomaly is different $\left(\theta \sim=-22^{\circ}\right.$ [四]). 


\section{Conclusion}

Keeping only the leading term in $1 / N$ at each order in the momentum expansion, we derived at $\mathscr{O}\left(p^{4}\right)$ a mass matrix in the flavor basis. The quantities are expressed in term of the masses and the ratio of decay constants $y$, instead of the low energy constants, allowing a clear understanding. Such a form allows the extraction of two parameters $\phi$ and $y$ from mass inputs. Their values are in agreement with the data.

The choice of the flavor basis is motivated the simple expression of the kinetic term and the diagonal matrix for the decay constants. Such features, not apparent in the $U(3)$ basis, make the calculation tedious in that basis and render the physics more obscure.

The properties of the flavor basis were identified and analyzed in the FKS scheme [四]. In this work, we relate this formalism with the chiral Lagrangian approach where no assumption on the mixing scheme for the decay constants is required. It is shown that the FKS ansatz is not compatible with the chiral Lagrangian. The difference stands in the parameter $\Lambda$. Taking the limit $\Lambda \rightarrow \infty$ renders the FKS scheme whereas its value, extracted from the data, is of the same magnitude as those of the other low-energy constants [2].

The predicted values (B.9) are in agreement with the data but leave a room for improvement since assumptions were invoked. Moreover, the glue content in the $\eta^{\prime}$ wave function should be properly considered at $\mathscr{O}\left(p^{4}\right)$. To this aim, the inclusion of the pseudoscalar glueball in the chiral Lagrangian in under construction [5]. At leading order, the inclusion of the glueball leads to a consistent mixing scheme for all decays [目].

\section{Acknowledgements}

This work has been partially funded by the Spanish Ministerio de Ciencia y Tecnología and UE FEDER under Contract No. FPA2007-65748 and by the Spanish Consolider Ingenio 2010 Program CPAN (CSD2007-00042). It is also partly funded by HadronPhysics2, a FP7-Integrating Activities and Infrastructure Program of the EU under Grant 227431.

\section{References}

[1] T. Feldmann, P. Kroll and B. Stech, Phys. Rev. D 58, 114006 (1998)

[2] J. M. Gerard and E. Kou, Phys. Lett. B 616, 85 (2005)

[3] V. Mathieu and V. Vento, Phys. Lett. B 688 (2010) 314

[4] C. Amsler et al. (Particle Data Group), Physics Letters B667, 1 (2008)

[5] V. Mathieu and V. Vento, Phys. Rev. D 81, 034004 (2010)

[6] C. Degrande, J. M. Gerard and V. Mathieu, in preparation 